\title{
On the applicability limits of some 'infinite' models in the course of electricity and magnetism
}

\author{
Vladimir Ivchenko*10 \\ ${ }^{1}$ Kherson State Maritime Academy, Department of Natural Sciences Training, Kherson 73000, Ukraine.
}

Received on March 19, 2021. Accepted on April 5, 2021.

\begin{abstract}
We present a quantitative analysis of some 'infinite' models, occurring in the course of electricity and magnetism. First, we estimate the deviations from the models of an ideal parallel-plate capacitor and solenoid arising due to the bending field effect. Next, we consider the models of an infinite wire and plane of uniform charge. We show that in the first case the real electric field differs from the idealized one because of the finite length of the wire. In the second case this difference is caused also by the non-uniform surface charge density. The issues outlined in this article will be useful for advanced undergraduates, studying solutions to electrostatic and magnetostatic problems.
\end{abstract}

Keywords: limit transition models, parallel-plate capacitor, solenoid, infinite charged wire, infinite charged plane.

\section{Introduction}

Ones of the most important limit transition abstractions [1] in the university course of electricity and magnetism are the so-called 'spatially infinite' models. These include the models of physical objects, whose dimensions in one or several directions are so large that, when solving a specific problem, the influence of edge effects, that is, the presence of edges in these directions, can be neglected. One can distinguish between two types of models of this kind:

1. The models of objects 'transverse' and 'longitudinal' sizes of which differ many times.

2. The models of objects, the distances to the edges of which from the observation points are much larger than the shortest distances from these points to the objects.

The first type includes, in particular, the models of an ideal parallel-plate capacitor and solenoid. As the examples of models of the second type we may mention the models of an charged infinite wire and plane.

In reference 20 a quantitative analysis of a model of the magnetic field of a long straight wire is presented. Here, we discuss the applicability limits of some other 'infinite' models in the course of electricity and magnetism. The correctness of the applicability of a particular model depends on the specific physical situation itself and, strictly speaking, should be determined by means of a physical experiment. However, for education purposes the accuracy of a model, i.e. the errors arising as a consequence of neglecting several factors considered

* Correspondence email address: reterty@gmail.com to be insignificant in the construction of the model can be estimated theoretically within the extended model taking into account these neglected factors. The issues outlined in this article will be useful for advanced undergraduates studying solutions to electrostatic and magnetostatic problems.

\section{An Ideal Parallel-plate Capacitor and Solenoid}

Ones of the basic models in the course of electricity and magnetism are an ideal parallel-plate capacitor and solenoid. Their "ideality" is caused by the fact that the field inside them is considered homogeneous everywhere. In fact, the field lines are bent at their edges (Figures 1 and 2).

Let us consider an idealized, parallel-plate capacitor with circular plates (for ease of comparison we choose "regular" shaped capacitor). Its capacitance is

$$
C_{0}=\frac{\epsilon_{0} \pi D^{2}}{4 d}
$$

where $\epsilon_{0}$ is the vacuum permittivity; $D$ is the plate diameter; $d$ is the plate separation. The approximate expression, taking into account the finite relative size of the plate, is known as Kirchoff's approximation [3] and can be represented in the following form:

$$
C \approx C_{0}+\frac{\epsilon_{0} D}{2}\left[\ln \left(\frac{8 \pi D}{d}\right)-1\right] .
$$

The detailed discussion of the derivation of Equation (2) is presented in the book by Sneddon [4. Here we briefly describe this process of derivation. The problem 


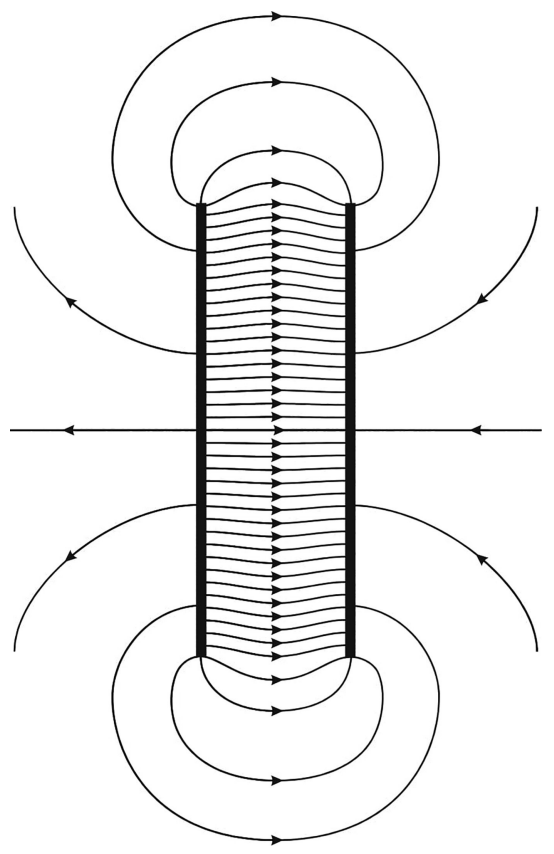

Figure 1: The field lines in the case of a real parallel-plate capacitor.

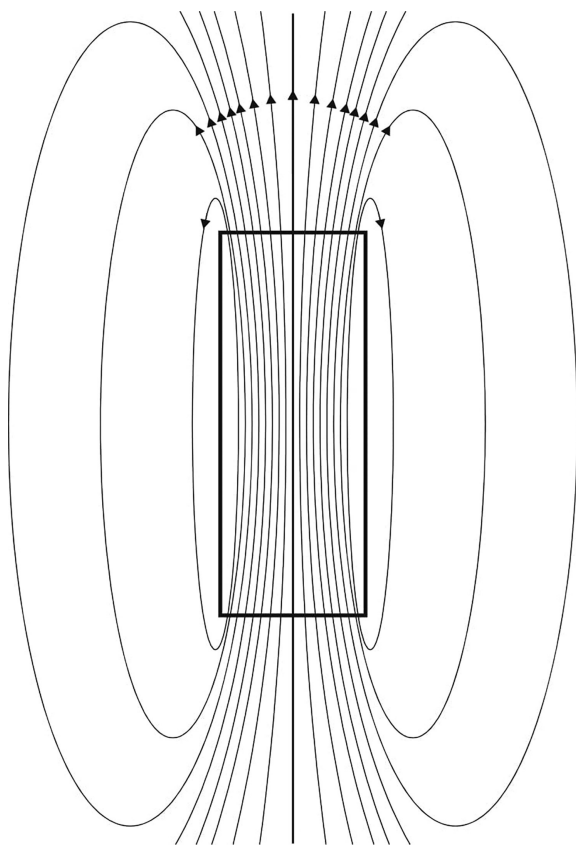

Figure 2: The field lines in the case of a real solenoid.

starts by solving the Laplace equation in the region between two charged discs having opposite potentials. From the condition that the partial derivative of the potential in the direction perpendicular to the disk surface is proportional near it to the surface charge density, one can find the radial distribution of the latter on the disk surface. This allows us to find further the total charge of one plate of the capacitor. As a result,

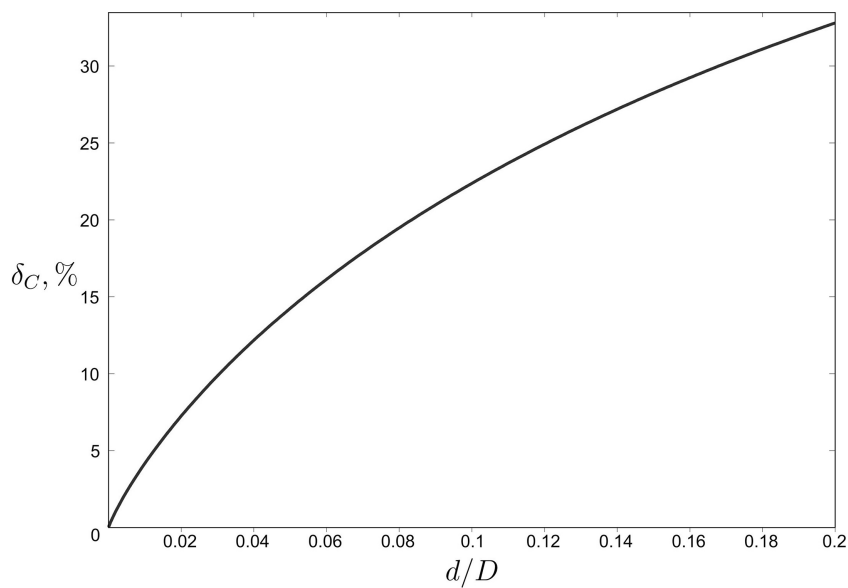

Figure 3: Relative error $\delta_{C}$ as a function of relative plate separation $d / D$.

the capacitance (the ratio between the charge and the potential difference) is presented in the integral form. This expression can be expanded in power series of the ratio $d / D$. It gives directly the Kirchoff's approximation.

According to Equation (2) $C>C_{0}$. This fact can be qualitatively understood as follows. The energy of a capacitor is equal to $C U^{2} / 2$. But for the real parallelplate capacitor of finite size there is an additional energy associated with the existence of the field outside it (see Figure 11. Then, at fixed voltage $U$, its accounting directly leads to some increasing of the capacitance.

In Figure 3 we plot relative error $\delta_{C}=\left(C-C_{0}\right) / C$ as a function of relative plate separation $d / D$. This error is not the error of calculations but appear as the result of difference between the simple and extended model. It is seen that the value of this quantity is equal to $5 \%$, if distance $d$ is a hundred times more than plate diameter $D$.

The inductance of an infinitely long cylindrical solenoid is given by

$$
L_{0}=\frac{\mu_{0} \pi n^{2} D^{2} l}{4},
$$

where $\mu_{0}$ is the magnetic constant; $n$ is the number of turns per unit length; $D$ is the coil diameter; $l$ is the length of the coil. Taking approximately into account the end-effect correction, the inductance of an finite length solenoid can be calculated as [5, p. 126]

$$
L \approx L_{0}\left(1-\frac{4 D}{3 \pi l}\right) .
$$

The derivation of Equation (4) can be easily done if we remember that the self-inductance is proportional to the free self-energy of the current. The latter can be calculated as a double integral over the surface of the solenoid [5]. Performing the approximate integration for $D \ll l$, we finally derive Equation (4).

According to Equation (4) $L<L_{0}$. This fact can be qualitatively understood as follows. The total magnetic 


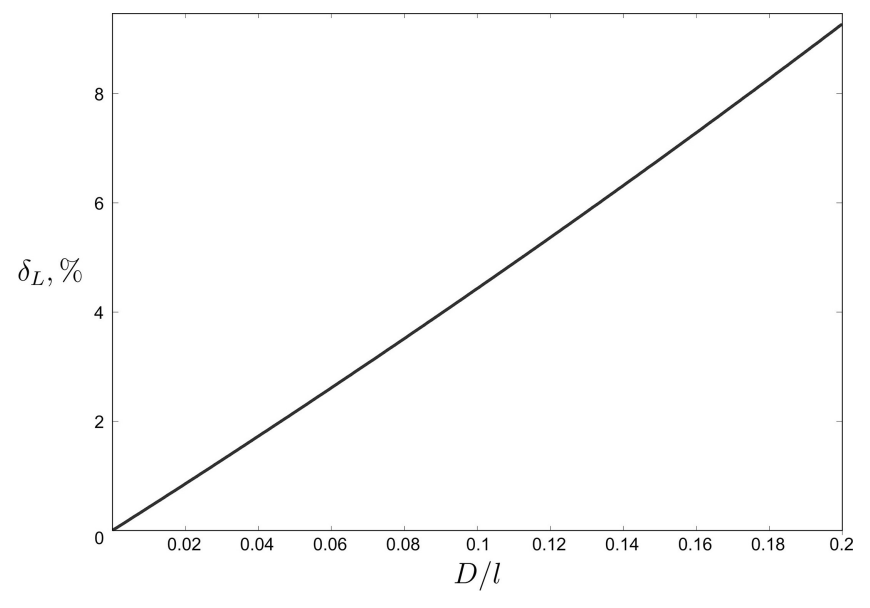

Figure 4: Relative error $\delta_{L}$ as a function of relative coil diameter $D / l$.

flux through the coil is obtained by adding this value for individual turns. But, at fixed value of current $I$, the magnetic flux through the peripheral turns decreases due to the weakening and bending of the magnetic field near the edges. This should directly lead to some decrease of the inductance.

In Figure 4 we plot relative error $\delta_{L}=\left(L_{0}-L\right) / L$ as a function of relative coil diameter $D / l$. It is seen that the value of this quantity is equal to $5 \%$, if length $l$ is a ten times more than diameter $D$.

\section{An Infinite Wire and Plane of Uniform Charge}

As the illustrative examples of the application of Gauss's theorem in the course of electrostatics, the calculations of fields of uniformly charged infinite wire and planes are given. The electric field of an infinite wire is

$$
E_{0}=\frac{\lambda}{2 \pi \varepsilon_{0} r},
$$

where $\lambda$ is the linear charge density; $r$ is the shortest distance from wire to the point of observation. The electric field of an infinite plane is given by

$$
E_{0}=\frac{\sigma}{2 \varepsilon_{0}}
$$

where $\sigma$ is the surface charge density. In both cases, the electric field is directed perpendicular to the object. Equations (5) and (6) are used for the approximations of the electric fields of a long conducting charged wire and disc. In reality, such objects are characterized by both non-uniform distribution of the surface charge density and finite size.

The exact expressions for the field potentials of the real conducting charged wire of finite size and circular disk can be found as the limiting cases of the potential of a conducting charged spheroid. For a line segment we have [5, p. 22]

$$
\begin{aligned}
\varphi= & E_{0} r \tanh ^{-1} \\
& {\left[\frac{l^{2} / 2}{r^{2}+z^{2}+\frac{l^{2}}{4}+\sqrt{\left(r^{2}+z^{2}-\frac{l^{2}}{4}\right)^{2}+l^{2} r^{2}}}\right]^{1 / 2}, }
\end{aligned}
$$

where the value of $E_{0}$ is defined by Equation (5); $l$ is the length of a wire; $r, z$ are the cylindrical coordinates of the point of observation (the origin coincides with the center of a line segment; $z$-axis coincides with a line segment; $r$-axis is perpendicular to a line segment). For a circular disk [5, p. 25]

$$
\begin{aligned}
\varphi & =E_{0} R \tan ^{-1} \\
& {\left[\frac{2 R^{2}}{r^{2}+z^{2}-R^{2}+\sqrt{\left(r^{2}+z^{2}-R^{2}\right)^{2}+4 R^{2} r^{2}}}\right]^{1 / 2}, }
\end{aligned}
$$

where the value of $E_{0}$ is defined by Equation $(6) ; R$ is the disc radius; $r, z$ are the cylindrical coordinates of the point of observation (the origin coincides with the center of a disk; $z$-axis lies in the plane of the disk; $r$-axis is perpendicular to the disk). In deriving Equation (8), we took into account that the disk has two identical surface areas. The derivation of the field of a conducting charged spheroid is based on using ellipsoidal coordinates and leads to the integral representation for the potential in form of an elliptic integral of the first kind [5, p. 22].

Equations (7) and (8) together with relations $E_{r}=$ $-\partial \varphi / \partial r$ and $E_{z}=-\partial \varphi / \partial z$ allow one to calculate relative errors

$$
\delta_{\vec{E}}=\frac{\sqrt{\left(E_{r}-E_{0}\right)^{2}+E_{z}^{2}}}{\sqrt{E_{r}^{2}+E_{z}^{2}}}
$$

in the determination of electric field $\vec{E}=\vec{E}_{r}+\vec{E}_{z}$, which arise in the case of replacing these bodies with the infinite and uniformly charged idealized objects. In Figures 5 and 6 , we present the results of the numerical calculation as contour lines, along which the relative error $\delta_{\vec{E}}$ is equal to $5 \%$. The domains corresponding to a larger value of this quantity are shaded in gray.

It can be observed that the maximum 'correct' distance from the wire to the observation points (about $15 \%$ of the wire length) corresponds to its middle, decreasing to zero as these points approach the edges of the wire. Since, the linear charge density of the wire turns out to be constant at such a limiting transition [6], the deviations from an infinite wire model are completely determined by the finite length of the wire.

In the case of a conducting disc the situation is somewhat different. The maximum 'correct' distance from the disk to the observation points (about $10 \%$ of the 


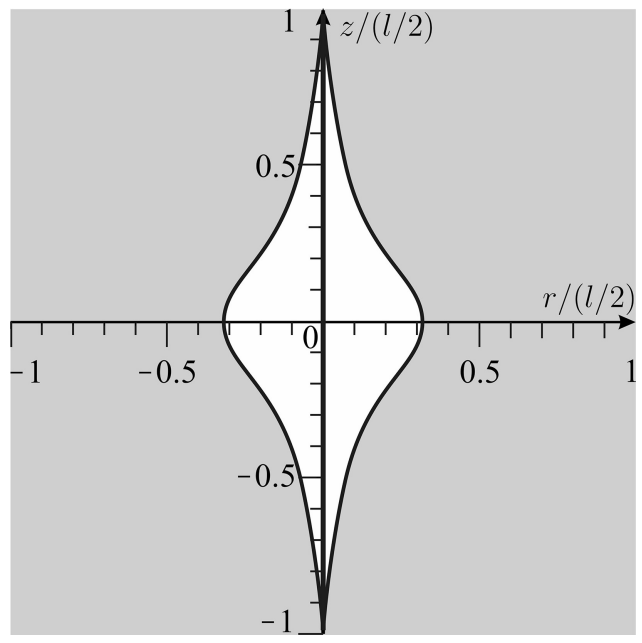

Figure 5: Domains of credibility (white) and incredibility (gray) of the infinite wire model for the line segment at $\delta_{\vec{E}}=5 \%$.

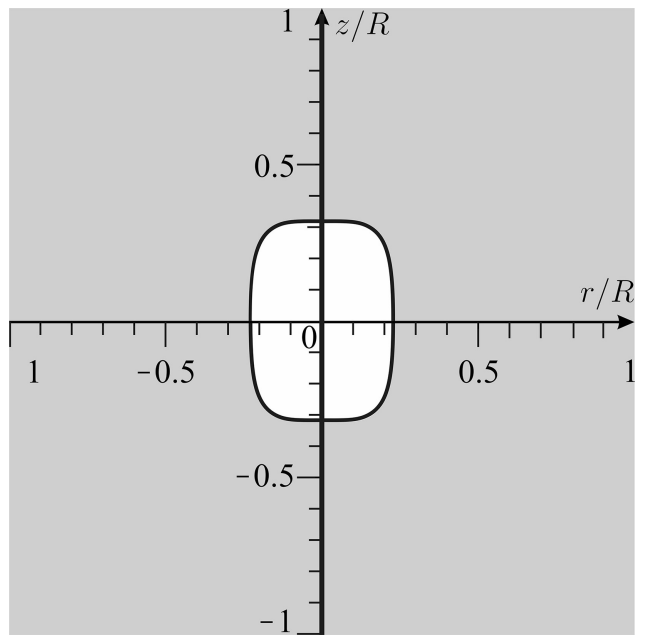

Figure 6: Domains of credibility (white) and incredibility (gray) of the infinite plane model for the disk at $\delta_{\vec{E}}=5 \%$.

disk diameter) also corresponds to its middle. However, due to the increase in surface charge density towards the edges of the disk, the dimension of the domain of credibility along the disk is much less than for the wire.

\section{Conclusions}

In this paper we present a quantitative analysis of the applicability limits of some 'infinite' models in the course of electricity and magnetism. We show that the real parallel-plate capacitor can be considered as the idealized one, if its longitudinal size is a hundred times more than the transverse size. The real solenoid can be seen as the 'infinite' one if this ratio is approximately equal to 10. Next, we discuss the influence of the non-uniform distribution of the surface charge density and finite size on the deviations from the models an infinite wire and plane of uniform charge. We visualize the errors of these two models and conclude that in the first case the real electric field differs from the idealized one because of the finite length of the wire. In the second case this difference is caused also by the non-uniform surface charge density. These findings can help readers to probe the limits of applicability of the considered models.

\section{References}

[1] V. Ivchenko, Eur. J. Phys. 39, 045004 (2018).

[2] V. Ivchenko, Eur. J. Phys. 41, 065201 (2020).

[3] A. Naini and M. Green, Am. J. Phys. 45, 877 (1977).

[4] I.N. Sneddon, Mixed boundary value problems in potential theory (Wiley, New York, 1966).

[5] L.D. Landau and E.M. Lifshitz, Electrodynamics of Continuous Media (Pergamon press, Oxford, 1984), 2nd ed.

[6] J.D. Jackson, Am. J. Phys. 68, 789 (2000). 